\title{
MENTAL SIMULATION FOR CREATING REALISTIC BEHAVIOR IN PHYSICAL SECURITY SYSTEMS SIMULATION
}

\author{
Volkan Ustun \\ Jeffrey S. Smith \\ Dept. of Industrial and Systems Engineering \\ Auburn University \\ Auburn, AL 36849, USA
}

\begin{abstract}
Mental simulation is proposed by cognitive psychologists as a candidate to model the human reasoning process. In this paper, we propose a methodology that models mental simulation to create realistic human behavior in simulated environments. This methodology is used to generate realistic intruder and guard behavior in physical security systems simulation. The behaviors include moving to a target while avoiding detection/capture for intruders and following and apprehending intruders for guards.
\end{abstract}

\section{INTRODUCTION}

Simulation models can be used to investigate real life situations in which human entities operate. In these types of models, it is important to model the behavior of human entities (or agents) close to their real life behavior. In real life problems, humans can make mistakes, their perceptions can be wrong, they can make awkward decisions, and they can come up with courses of action that are different from the courses of action generated based on extensive analysis. As a result, human behavior shows significant variability in real life settings. These tendencies are often exacerbated in the presence of stress. Therefore, realistic and credible simulations of real life situations require capturing the variability in agent movements and behavior.

Several researchers in cognitive psychology agree upon the fact that the human decision making process is not outcome oriented in real life situations. Instead of listing and comparing relevant action alternatives, humans first rely on their intuitions and the process they employ to generate actions. The commonly accepted two-system view approach also highlights this phenomenon (Stanovich and West 2000; Kahneman 2002). The two-system view defines two types of cognitive processes; intuition and reasoning. Intuition is evoked and it generates a response particular to the situation at hand. Reasoning is then used to either evaluate the response generated by the intuition process or to perform effortful reasoning if there is no intuitive response.

The intuitive response can be in the form of a sequence of actions that has the potential to achieve a goal that an agent has. Assuming that a response is generated by the intuition cognitive process, there is a need for a mechanism to evaluate this response. The reasoning process is responsible for performing this evaluation and several psychologists propose simulation or the "simulation theory" in their terminology as a candidate to model the human reasoning process when social rationality, which is to understand that another agent may have goals and expectations different from one's own, is a concern (Davies and Stone 1996). The simulation theory is based on the capability of the human brain to simulate in order to predict and understand an action by mentally processing its production in one's own mind (Stich and Nichols 1995). The simulation theory is situated on the fact that human brains and thinking processes are similar and hence if one can make adjustments for relevant differences such as situation or emotional state then he/she can use his/her mind to simulate mental processes of others in order to describe the other's behavior and furthermore predict these behaviors. However, it is important to underline the fact that putting yourself in other's shoes is to project yourself into other's situation but not to attempt to project yourself into the other's mind. It is not the same as deciding what I myself would do but to try to make adjustments for relevant differences. Simulation is used cooperatively as well as competitively: bridge players project themselves into their partner's shoes whereas chess players project themselves into their competitor's shoes.

The simulation theory has been supported by the studies of several researchers from different fields. Kahneman and Tversky (1982) defined a heuristic named "simulation heuristic" based on their observations in laboratory settings. In this heuristic, they described how a person might build a simulation to explain how something might happen; if the simulation required too many unlikely events, the person would judge that thing to be implausible. Natu- 


\section{Ustun and Smith}

ralistic Decision Making (NDM) researchers also have an interest in simulation theory. Gary Klein, who is one of the founders of NDM research states that simulation theory or "mental simulation" as he names it, is central to decision making. He observed that people construct mental simulations almost the way one builds a machine (Klein and Crandall 1995). Klein (1998) defines mental simulation as:

"Mental simulation is the ability to imagine people and objects consciously and to transform those people and objects through several transitions, finally picturing them in a different way than at the start."

"Look-ahead simulation" has also been applied in the real-time control and scheduling of flexible manufacturing systems. Wu and Wysk (1989) developed a discrete-event simulation based scheduling mechanism to evaluate the performance of different dispatching rules in a flexible manufacturing cell for a short planning horizon. Smith et al. (1994) applied discrete-event simulation for shop floor control for a flexible manufacturing system. However, look-ahead simulations are executed centrally with complete information on the current state of the system. The simulation methodology proposed in this paper requires actual realism; the agents cannot use the information that they have not perceived. This is also different from the general approach used in computer/video games in which the objective is to increase the realism perceived by the users. Action generation scripts in computer/video games can include "cheats" to collect information on human opponents and this information can then be used to create actions that are perceived as intelligent by the users (Bourg and Seeman 2004). To rectify this limitation, we are creating a mental representation of the environment for each agent based on their perceptions. This mental representation is then used in running mental simulations to evaluate a course of action. Therefore, there is a need for formally defining the rules to generate this mental representation. Each agent needs to perceive and to store information on the objects and other agents in the environment. Furthermore, each agent has a personality that affects the evaluation process of mental simulations.

While presenting aforementioned concepts, physical security systems simulation is used to provide examples. Physical security systems simulation models are used to investigate the system performance in settings which resemble real life situations. Use of discrete-event simulation as a tool to assess the vulnerability of fixed-site facilities has been discussed by Jordan et al. (1998) and Smith et al. (1999). However, these simulation models use predefined entity routes and rules of behavior to inject variability into the models that simulate real life situations. A more recent conceptual discussion on modeling agent behavior for a realistic environment is provided by Ustun et al. (2006).
The initial focus in this paper is given to the mental representation of the environment for the agents. The information acquisition process that the agents use to build up information about the environment and other agents builds up on the mental representation discussion. Execution of mental simulations is then discussed with a demonstration of the concept. The paper concludes with a discussion on the current status of this research and future works.

\section{MENTAL REPRESENTATION OF THE ENVIRONMENT}

A conceptual data model named Hierarchical Graph Representation for Scenes (HIGHRES) is extensively discussed in an earlier paper (Ustun et al. 2005). This paper describes the conceptualization of the static aspects of a facility such as geometry, structure etc. and defines the relations between these aspects and the active entities in the simulation of physical security systems. HIGHRES defines three specific data types: solid objects, zones, and portals. A solid object is a solid shape; whereas a zone is a volume. Portals are objects that connect two or more zones. There are also two graph constructs to capture the interactions between the spatial features and the agents. The Zone Movement Graph presents the possible movements between zones and the Portal Visibility Graph shows the portals visible from a given portal.

The mental representation of the environment is based on the HIGHRES model. This representation is comprised of beliefs, which correspond to the information the agent has about the environment. In other words, beliefs are assimilated information and conclusions that the agent believes to be true. However, the beliefs can be inaccurate or partial in realistic environments. Therefore, there is a need for a mechanism that differentiates between the real world and the agents' worlds. Each agent stores a mental representation of the environment, which is updated each time the agent's beliefs are updated. This mental representation is then used while analyzing any possible course of action. In order to construct a realistic mental representation of the environment, an agent needs to construct beliefs on: (1) static objects in the environment, and (2) other agents in the environment.

\subsection{Beliefs on static objects}

The beliefs on the static objects in the environment are represented with the type of the objects and the coordinates of the objects. At the initialization stage, instantiation of each agent's knowledge base includes information about 3 different types of objects of the HIGHRES model: zones, portals, and solid objects. In addition to the HIGHRES objects, sensors are also of importance to the agents in a physical security system and hence, they need to be represented in the agents' beliefs. Each agent's knowledge 


\section{Ustun and Smith}

base is created separately so the following discussion is applied to each agent separately.

Different cases are defined to describe agent's information on HIGHRES data types:

- The agent has full coordinate information on the portal/solid object/zone,

- The agent assumes that there is a portal/solid object that is not actually in the environment.

- The agent does not know about the portal/solid object/zone,

- The agent knows about the portal/solid object/zone but doesn't have any information about the coordinates,

- The agent has wrong coordinate information on the portal / solid object.

Information on sensors is also defined in a similar fashion. There are three cases while defining the sensors:

- The agent has full coordinate and parameter information on the sensor,

- The agent assumes that there is sensor that is not actually in the environment,

- The agent does not know about the sensor.

Parameters of the sensors represent the capabilities of the sensor. For example, a surveillance camera is defined by the look direction, the range and the half-cone angle in addition to the coordinates of the sensor. The inaccurate information on sensors is represented by using a sensor that is not actually in the environment. For example, if the agent has inaccurate information on the look direction of a surveillance camera, this will be represented as a nonexistent surveillance camera in the agent's mental representation. This belief can later be updated when the agent discovers the existent camera.

The beliefs on static objects are captured in the agent's mental representation using two graph constructs: (1) the Zone Movement Graph and (2) the In-Zone Graphs. Zone Movement Graphs represent the associations between the zones in the facility. The In-Zone graphs provide a cellular decomposition of the zone and each cell is marked based on whether any solid object obstructs movement in the cell. In addition, the In-Zone Graphs can include information on the desirability of the cell. For instance, a cell that is visible to a surveillance camera would be less desirable for an intruder. Each zone has an associated In-Zone Graph and this In-Zone Graph is used in generating/analyzing movements within a zone. Zone Movement Graphs are constructed using the information on zones and portals. InZone Graphs are constructed using information on the zone, the solid objects and sensors included in the zone and the portals that are bounding the zone. These graphs are constructed for each agent using the cases defined above for each static object.

\subsection{Beliefs on Agents}

Agents hold beliefs about other agents in the environment and these beliefs are then employed in the mental representation of the environment. There are three types of beliefs on other agents:

- Positions of other agents,

- Activities of other agents,

- Multiple step activities such as movement,

- Single step activities such as picking up item.

- Types of other agents.

An agent needs to know about the positions and activities of other agents in the environment in order to project the other agents' anticipated movements. In other words, the agents should have the capability of putting themselves in other agents' shoes. The primary difference between single step and multiple step activities is that the agent needs multiple perceptions to construct beliefs on multiple step activities of other agents. One example for multiple step activity is movement. In order to construct beliefs such as the other agent is moving in a certain direction or the other agents is patrolling in the facility on a certain route, the agent needs to recognize the agent of concern multiple times at different positions.

\section{INFORMATION ACQUISITION}

Vision is the primary perception mechanism used by the agents. The vision mechanism is modeled by employing visual cues; a type of sensory cue. A sensory cue is basically a signal that can be extracted from the sensory input by a perceiver. Any object or agent is defined by a set of points and the sensory input is the set of points that define the object or the agent. Line-Of-Sight calculations discussed in Ustun et al. (2005) are performed using these points and the points that are visible to the agent are regarded as visual cues. The agent then uses a recognition function to determine whether the agent recognizes the individual objects, the agents or the actions of the agents using the visual cues.

The sensory input for an object is comprised of the eight points that define the object in the HIGHRES model plus the center point of the object. Similarly, the sensory input for an agent is composed of six points: one for the head, one for the torso, two for the hands and two for the feet. If an object or an agent is of any interest, Line-ofSight calculations are performed and the points that are visible from the sensory input are fed to the recognition function. Currently, two types of recognition functions are defined. Each object, agent or process that might be of in- 


\section{Ustun and Smith}

terest should be associated to a recognition function. The first recognition function is based strictly on the number of visual cues - if there is certain number of visual cues then the subject is recognized. The second type of recognition function differentiates between the visual cues. For example, points representing the hands of an agent in addition to other possible points need to be seen in order to recognize the fact that the agent is concealing an item. In addition, activity detection requires human comprehension to correctly interpret the activities happening in the environment. For example, Tickner and Poulton (1973) discuss the factors that affect the detection probability of humans on certain activities from surveillance video streams. Important factors in detecting an activity are:

- Distance of the location of the activity to the camera (or looking point), closer to the activity better detection,

- Duration of the activity, longer the activity better detection,

- Hours worked by the guard -- the fewer hours worked by the guard, the better the detection probability,

- Number of monitors being watched -- the more monitors being watched, the less probable the detection.

The aforementioned beliefs are constructed by employing the perception/recognition mechanisms. At any point in time, agents collect position information about other agents and objects of interest. Furthermore, the agent activities of importance defined by the application domain are also perceived.

Information acquisition is a computationally intensive task. Therefore, it is necessary to define and use a computationally efficient information acquisition process. One way to do this is only triggering the perception mechanism of the agents either when there is something interesting happening or when there is potential to discover new objects or to update beliefs that are inaccurate instead of running the perception mechanism continuously. The Portal Visibility Graph is the primary tool to limit the number of objects that need to be checked. As stated previously, the Portal Visibility Graph stores the information on which other portals are potentially visible from a given portal. The purpose here is to find the zones that the agent can potentially see and then run the perception mechanism only for the objects and agents that are in the potentially visible zones at the time. The basic algorithm used for this purpose is as follows:

1. Find all the portals that are bounding the zone that the agent is in.

2. Find the portals that are visible to the agent from the portal list found in Step 1 (this step incorpo- rates the details on the current position of the agent, the agent's looking parameters that are look direction, range, and cone angle).

3. Find all the portals that are potentially visible from the portals found in Step 2.

4. Find all the zones that are bounded by any of the portals found in Step 3.

The algorithm presented above finds all the zones that are potentially visible to the agent. After finding all the potentially visible zones, it is necessary to find all the objects in these zones that either the agent does not know about or has inaccurate information about in the belief set. Perception/recognition process is then executed and the necessary updates are performed on the belief set for the objects recognized.

Information acquisition on the position of other agents of interest is run in a similar fashion. For the agents of interest in potentially visible zones, perception/recognition process is executed and the belief set on the agents is updated using the results of the perception/recognition process. It is important that the type of the agent is not always revealed to other agents. For example, a guard may not differentiate between intruders and neutrals unless a significant activity is performed by the intruder. Therefore, the type check and hence belief formation is only possible if the agent knows the type of the agent of interest.

The next fundamental question is how frequently is the perception/recognition process is triggered. The quick answer is each time the agent moves to a new cell in the In-Zone Graph of the associated zone. This approach will provide the necessary precision for the information acquisition process.

The information acquisition on the activities of the other agents is triggered separately. When an agent performs an interesting activity, the perception/recognition process of all the agents that are in the potentially visible zones at the time and that are interested in the perceiving this activity is triggered. Interesting activities are application specific and details on interesting activities for a retail store security example is provided in Ustun et al. (2006).

\section{RUNNING MENTAL SIMULATIONS}

The course of action devised by a method (e.g. a heuristic) is comprised of a sequence of activities that the agent is projecting to perform. In most physical security cases, the activities only involve movements and physical activities such as picking up items, opening doors, monitoring surveillance camera streams, etc. After generating a sequence of activities, the agent needs to further analyze the potential to achieve the goal. Considering the stochastic nature of some of these activities in addition to the necessity of taking into account other agents' activities, the agent needs to look into a set of possible sample paths. As stated earli- 
er, mental simulation is the process that is used by humans in performing the projection of future activities and assessing the likelihood of success in achieving the goal. Mental simulations require predicting the behavior of other agents, which requires projecting the agent into other's situation. For example, if an intruder uses force to open a door such that it is punctured, a guard that perceives the punctured door would likely check the situation as the punctured door would be unusual. Therefore, mental simulations should be capable of modeling reasonable behavior on other agents' part. However, if the intruder opens the door with a key and leaves the door closed, the expected behavior on the guard would likely not involve checking the situation.

Mental simulations are run using the beliefs of the agent. Therefore, only the objects that are known to the agent with the known parameters are used in running the simulations. Anticipation of other agents' behavior is primarily generated by using the beliefs on other agents, which are discussed in the information acquisition process. The primary output metric of the mental simulations is the number of successful replications in achieving the goal. Mental simulations are run for a certain number of replications and the output is used in making the decision whether to endorse the course of action as it is. The approach taken at this point is similar to the two-system view (Stanovich and West 2000; Kahneman 2002). There are three cases defined in the two-system view; (1) direct endorsement of the course action, (2) using the course of action as an anchor for adjustments, and (3) rejecting the course of action. This decision is made by comparing the agent's degree of risk seeking by the result of mental simulation. The degree of risk seeking in course of action assessment, which is part of the agent personality definition, has two parameters: (1) direct endorsement and (2) accepting as an anchor. Therefore, if the probability of success, which is the result of the mental simulation run, is greater than the first parameter, the course of action is endorsed and implemented. If it is greater than the second parameter but less than the first parameter, the course of action is used as anchor to perform modifications that potentially increases the probability of success. Otherwise, the agent tries to find another heuristic that hopefully better fits the current situation.

When the agent chooses to make adjustments using the course of action at hand as an anchor, the agent first identifies the activities that are potentially problematic. Based on this assessment, the agent applies an operator to the course of action used as an anchor. Some of the example operators in physical security systems are:

- Wait: The agent waits at one of the points that is visited,

- Hide: The agent tries to hide behind a solid object in the facility,
- Add, remove, change zone: The agent changes one of the zones visited, or adds a new zone to visit, or removes a zone from the course of action.

An example for this adjustment process is given next. In this configuration, there are two surveillance cameras and a security guard in a retail store. There is one intruder at the entrance of the retail store. The goal of the intruder is to get an item from the second aisle on the left. In order to achieve the goal successfully, the intruder should not be seen while picking up this item. The intruder believes that there are two surveillance cameras in the store. In addition, the intruder believes that the security guard is patrolling in the store and the security guard is currently at the top right corner of the retail store. This situation is depicted in Figure 1.

Let's assume that the intruder agent developed a course of action using a heuristic that finds the shortest path to target point. This course of action is demonstrated in Figure 1. At time 1, the intruder is at the door. At time 2, the intruder gets out of the entrance area. At the time 3, the intruder arrives at the lower end of the second aisle from the left and at time 4, the intruder arrives at the target point where the intruder picks up the item. Based on this course of action, the intruder agent can run mental simulations (or generate sample paths) to further investigate the situation. Based on the beliefs of the agent, the security guard is moving from right to left with a certain speed, which might differ in different replications. One of the possible sample paths for the security guard's movement is also depicted in Figure 1. Based on this mental simulation, the intruder is not visible - the views of both surveillance cameras are obstructed by the shelves - to surveillance cameras while picking up the item if the intruder's beliefs on the surveillance camera locations are correct. However, the intruder assesses that there is a possibility that it is seen by the guard while picking up the item.

Assuming that the number of successful replications is less than the first degree of risk seeking parameter but greater than the second one, the agent performs a modification using the current course of action as an anchor. In this modification, the agent simple adds the fourth zone from the left to its path. This new situation is presented in Figure 2. Mental simulation process is rerun and if the number of successful replications is greater than the first degree of risk seeking parameter, the agent implements the modified course of action.

The agent generates expectancies while running the mental simulations based on the successful replications. There are two types of expectancies that are generated by the agents:

- Position of the agent at time $t$,

- Position of other agents at time t. 


\section{Ustun and Smith}

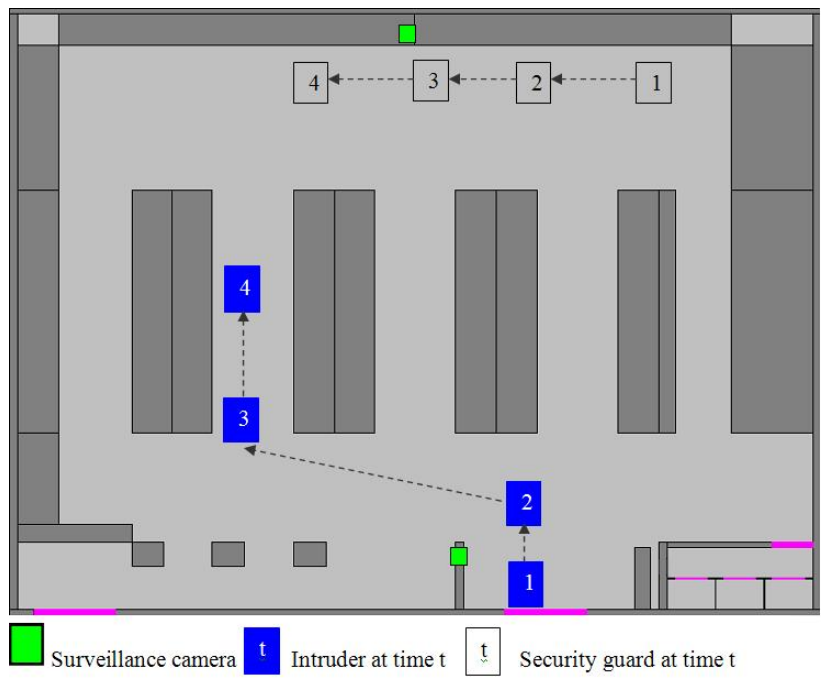

Figure 1: Initial Course of Action

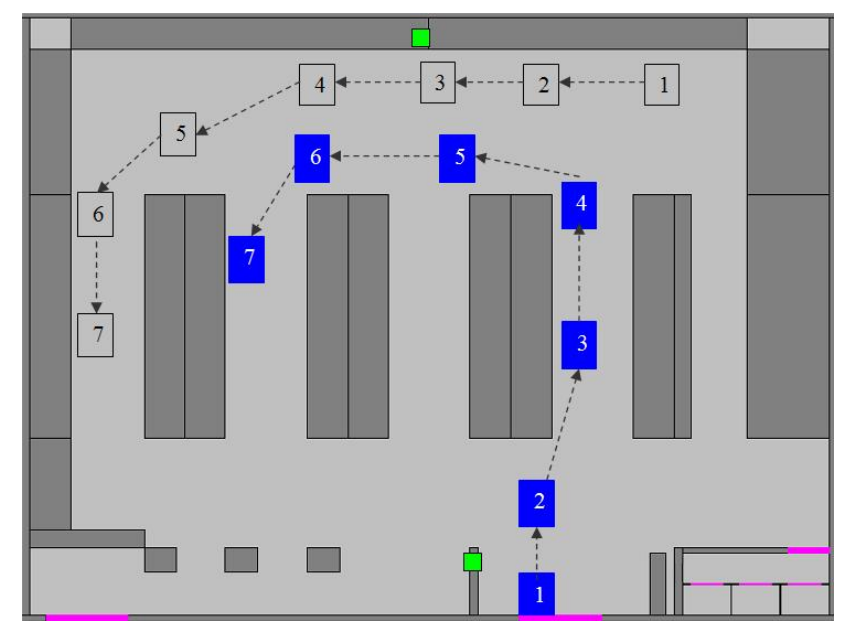

Figure 2: Course of Action Adjustment

The reason for expectancy generation is that actual implementation of the course of action might be different than the projection. Therefore, the agent needs a mechanism to detect deviations from the situations occurring in the mental simulation. Another parameter that defines the personality of an agent is the boldness. When a deviation from the expectancy occurs, the agent needs to make a decision on whether to reconsider the current course of action. The bolder the agent is the more deviation from expectancy the agent can tolerate. If the situation requires reconsideration, the agent first checks whether the goal is still achievable. If the goal is not achievable, the agent needs to drop the goal. Otherwise, the agent updates the timing information on the current course of action and runs a certain number of mental simulation replications to check whether the course of action is still acceptable. If this is the case, the agent con- tinues with the updated course of action. If not, the agent needs to devise a new course of action.

Acquisition of new information on static objects also needs to be taken into consideration. Assume that the intruder has discovered that there is a pallet in the second aisle from the left at time 6 (based on the course of action depicted in Figure 2). The mental representation of the intruder on the static objects at time 6 is presented using a InZone Graph construct in Figure 3. In this figure, the red cells represent the obstruction of movement by static objects, yellow cells are the cells seen by the surveillance cameras, and the gray cells are the cells that the intruder can go based on the intruder's beliefs on the object locations and the look direction of the surveillance cameras. The pallet blocks the original path devised for moving between locations at time 6 and at time 7. Therefore, the agent needs generate a new path that turns the corner around the pallet. This new course of action needs to be evaluated again by running mental simulations.

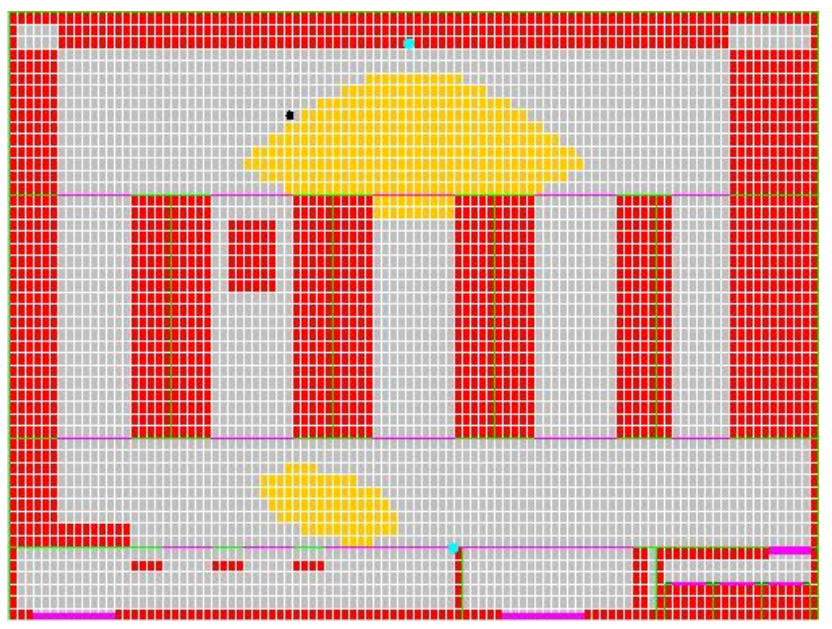

Figure 3: Intruder's mental representation at time 6

\section{CONCLUSION}

This paper presents a methodology for running mental simulations in modeling the human reasoning process. The methodology uses a course of action generated by the agent as an input. For the purposes of this paper, we have assumed that the agent has a set of heuristics available. The personality and the experience of the agent determine the heuristic selected that fits the most to the situation as it is perceived. The agent then uses the selected heuristic to generate a course of action and mental simulations are used to evaluate the course of action as described in this paper.

The set of heuristics available to the agent is highly dependent on the application domain. Currently, we are working on developing some realistic scenarios for physical security systems for operational validation of our methodology. The operational validation process involves va- 
lidating the realism of the methodology; human subjects will be asked whether the behavior that the agents perform is reasonable given the agent's goals and beliefs. We are waiting for the operational validation to be completed before going further in the implementation of realistic cases and hence, the discussion in this paper is rather at a conceptual level.

Physical security systems used in banks, retail stores, and buildings are possible examples that we focus for implementation. However, the described methodology is quite generic and it can be plugged into a wide variety of simulation models such as simulation-based games and evacuation models that use human entities and are particularly concerned with the interactions of these entities.

\section{REFERENCES}

Bourg D.M. and G. Seaman. 2004. AI for Game Developers. O’Reilly Media.

Davies M. and T. Stone. 1995. Folk Psychology: The Theory of Mind Debate. Blackwell Publishers.

Jordan S.E., M.K. Snell, M.M. Madsen, J.S. Smith, and B.A. Peters. 1998. Discrete-event simulation for the design and evaluation of physical protection systems. In Proceedings of the 1998 Winter Simulation Conference, ed. D.J. Medeiros, E.F. Watson, J.S. Carson, and M.S. Manivannan, 899-905. Piscataway, New Jersey: Institute of Electrical and Electronics Engineers.

Kahneman D. and A. Tversky. 1982. The Simulation Heuristic. In Judgment under Uncertainty: Heuristics and Biases, ed. D. Kahneman, P. Slovic, and A. Tversky. Cambridge University Press.

Kahneman D. 2002. Maps of Bounded Rationality: A Perspective on Intuitive Judgment and Choice. Nobel Prize Lecture.

Klein G. and Crandall B.W. 1995. The Role of Mental Simulation in Problem Solving and Decision Making. In Local Applications of the Ecological Approach to Human Machine System, ed. P. Hancock, J. Flach, J. Caird, and K. Vincente, 325-358. Lawrence Erlbaum Associates.

Klein G. 1998. Sources of Power. The MIT Press.

Smith J.S., R.A. Wysk, D.T. Sturrock, S.E. Ramaswamy, G.D. Smith, and S.B. Joshi. 1994. Discrete-Event Simulation for Shop Floor Control. In Proceedings of the 1994 Winter Simulation Conference, ed. J.D. Tew, S. Manivannan, D. A. Sadowski, and A. F. Seila, 962969. Piscataway, New Jersey: Institute of Electrical and Electronics Engineers.

Smith J.S., B.A. Peters, S.E. Jordan, and M.K. Snell. 1999. Distributed real-time simulation for intruder detection system analysis. In Proceedings of the 1999 Winter Simulation Conference, ed. P.A. Farrington, H.B. Nembhard, D.T. Sturrock, and G.W. Evans,
1168-1173. Piscataway, New Jersey: Institute of Electrical and Electronics Engineers.

Stanovich K.E. and R.F. West. 2000. Individual Differences in Reasoning: Implications for the Rationality Debate. Behavioral and Brain Sciences 23:645-665.

Stich S. and S. Nichols. 1995. Simulation or Tacit Theory. In Folk Psychology: The Theory of Mind Debate, ed. M. Davies and T. Stone. Blackwell Publishers.

Tickner A.H. and E.C. Poulton. 1973. Monitoring up to 16 Synthetic Television Pictures Showing a Great Deal of Movement. Ergonomics 16:381-401.

Ustun, V., H. Yapicioglu, S. Gupta, A. Ramesh, and J.S. Smith. 2005. A Conceptual Architecture for Static Features in Physical Security Simulations. In Proceedings of the 2005 Winter Simulation Conference, ed. M. E. Kuhl, N. M. Steiger, F. B. Armstrong, and J. A. Joines, 345-352. Piscataway, New Jersey: Institute of Electrical and Electronics Engineers.

Ustun V., L. Yilmaz, and J.S. Smith. 2006. A Conceptual Model for Agent-based Simulation of Physical Security Systems. In Proceedings of the 2006 ACMSE Conference, 365-370.

Wu D.S. and R.A. Wysk. 1989. An Application of Discrete-Event Simulation to On-line Control and Scheduling in Flexible Manufacturing. International Journal of Production Research 27 (9): 1603-1623.

\section{AUTHOR BIOGRAPHIES}

VOLKAN USTUN is a Ph.D. candidate in the Industrial \& Systems Engineering department at Auburn University. He received his B.S. and M.S. degrees in Industrial Engineering from Middle East Technical University (METU), Turkey in 1997 and 2000, respectively. Prior to joining the Ph.D. program at Auburn University, he has worked as a software engineer at The Scientific and Technical Research Council of Turkey (TUBITAK). Volkan Ustun is a member of IIE and INFORMS. His email address is $<$ ustunvo@auburn. edu>.

JEFFREY S. SMITH is a professor in the Industrial \& Systems Engineering department at Auburn University. Prior to joining Auburn, Dr. Smith was an associate professor in the Industrial Engineering Department at Texas A\&M University. He received the B.S. in Industrial Engineering from Auburn University in 1986 and the M.S. and Ph.D. degrees in Industrial Engineering from Penn State University in 1990 and 1992, respectively. In addition to his academic positions, Dr. Smith has held industrial positions at Electronic Data Systems and Philip Morris U.S.A. Dr. Smith is an active member of IIE and INFORMS. His email and web addresses are <jsmith@auburn.edu> and $<$ http: / / sim.auburn.edu/ jsmith $>$ 